\title{
Analysis of Key Technical Issues of Electronic Communication Systems
}

\author{
Wu Lan, Chen Yarong, Guo Jianxin and Chu Peng \\ The College of Information Engineering, Xijing University,710123
}

Keywords: Electronic Communication System, Mobile Communication, Satellite Communication

\begin{abstract}
Electronic communication technology determines people's living heights and affects people's living habits and working methods. With the development of electronic communication technology, people are enjoying the convenience it brings, such as communication anywhere and anytime, and 360-degree video calls, enabling long-distance communication. In addition to affecting people's lifestyles, electronic communication also determines a country's international status and level of economic development. At present, China still has a lot of room for development in electronic communication, but at the same time, there are still many problems. This paper mainly analyzes the typical examples of key technical issues of electronic communication systems, so as to better meet the needs of people's lives in the future.
\end{abstract}

\section{Introduction}

Nowadays, with the rapid development of the world, economic, cultural and political integration is increasingly being promoted by the public. The gap between the rich and the poor in countries around the world is gradually decreasing, and the information exchange between countries is becoming faster and faster. In the past, the situation in which people did not care about things that were not related to themselves did not exist anymore, and the information exchange zero time difference was truly achieved. The speed at which people accept new things is also accelerating, and people's knowledge reserves are also increasing. In today's fast-paced development, people have long been unable to adapt to the era when the previous days are slowly for everything. It is the electronic communication system and its derivatives that change people's lives. The application of electronic communication systems in people's lives is quiet and infiltrated little by little.

The use of electronic communication systems in people's lives has accelerated the speed at which people learn new knowledge and new things, and has also promoted the development of the information industry. It still can be remembered that in the 1960s and 1970s, when black-and-white televisions, radios, and pagers appeared in people's lives, everyone's eyes were full of doubts. No one believed in that people can see the dynamic characters from this little black box, people can hear the familiar family and people need not go back tens of kilometers as before just to call someone back. People have gradually accepted electronic communication technology, and people have gradually paid attention to electronic communication systems. Therefore, in the 21 st century of rapid development, it is our unshirkable duty to increase research on electronic communication systems and promote technological innovation in the electronic communication industry.

At present, electronic communication systems have been widely used in various fields of people's lives and learning. In the process of development, we have also found many problems. Especially in the analysis of key technical issues in electronic communication systems, everyone has their own views. Therefore, in the process of its development, it has been deeply and comprehensively explored in various aspects in order to deal with it in the future using scientific and technical methods.

\section{Overview of Electronic Communication Systems}

Generally, all systems capable of performing information transmission tasks can be collectively referred to as communication systems [1]. According to whether the baseband signal transmitted in the channel is an analog signal or a digital signal, the communication system can be divided into two types, namely, an analog communication system and a digital communication system [2]. 


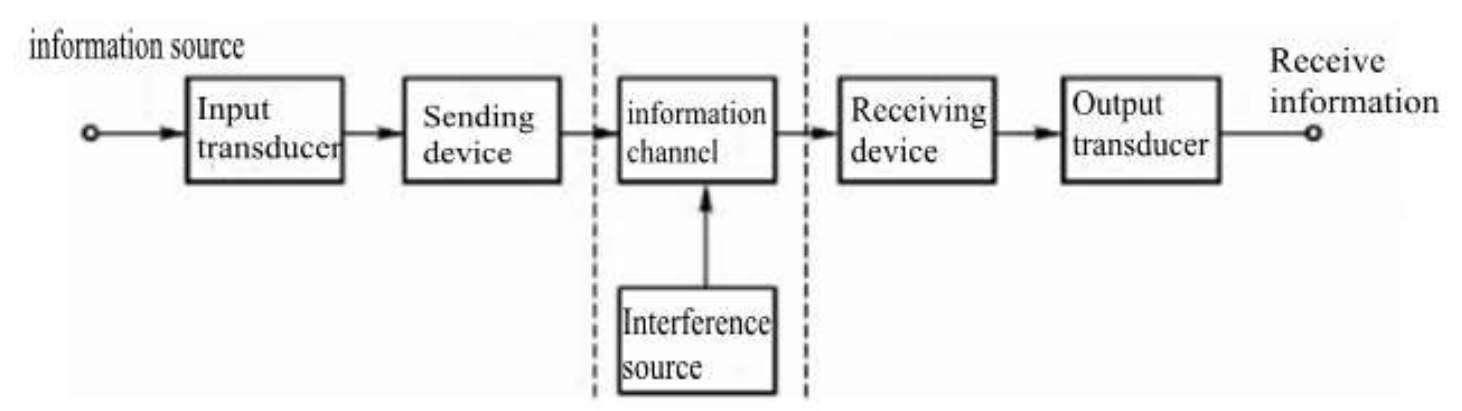

Figure 1. Communication system composition

Prior to the 1970s, some of the functional circuits in communication systems were generally constructed of discrete component circuits. With the rapid development of science and technology, integrated power supplies, operational amplifiers, low-frequency power amplifiers, and various frequency amplifiers and other integrated devices have been widely used in the late 1970s [3]. These general-purpose monolithic integrated devices, together with a small number of external components, form a separate subsystem, which can realize functions such as voltage regulation, amplification active filtering, modulation and demodulation, etc. If a variety of chips are combined, a complete electronic system can be formed.

With the passage of time, people's daily lives have also changed. The communication system has also developed into an electronic communication system, which plays an increasingly important role in people's study and lives. It not only improves people's living standards, but also makes China's social and economic development a step forward. And with the advancement of technology, people have taken more advanced ideas and humanized designs into consideration, serving people's daily lives, thus promoting the application of electronic communication systems in people's lives and learning.

Today, electronic communication technology has evolved into a modern communication technology and has become an important part of it. Secondly, electronic communication technology is also an important foundation of the information society, an important part of high technology, and even determines the economic level of a country and a nation. Now, we are in a global information integration environment. Electronic communication technology is always around us, and it comes in all aspects, including mobile phones, television, radar speed, sonar exploration, navigation direction, and other fields. [4], as well as military development and information systems in all sectors of the national economy will use electronic communication technology.

The most typical practical application examples in electronic communication systems are mobile communications and satellite communications. Their practical applications have achieved good results in China and even in countries all over the world. Among them, mobile communication includes satellite communication, cellular systems, cluster systems, radio paging systems and other fields [5].

\section{Key Technical Issues of Electronic Communication Systems}

Today, electronic communication has long been integrated into people's lives, and can not be separated like the air. Interpersonal communication has never been timely, not fast, and is available anytime and anywhere. While enjoying the convenience it brings, it will also create more production value and wealth, accumulate more wealth, and thus promote the development of the entire national economy. At present, competition among many countries is in the high-tech industries involving national security, such as electronic communication technology and defense electronic security systems, satellite stations, etc., which has become a fundamental symbol of a country's soft power.

In recent years, electronic communication technology has been widely used. The most representative of these are mobile communications and satellite communications, but they still have 
many technical issues that need to be addressed in order to better serve people's lives. The practical application of mobile communication technology is embodied in various electronic products in people's lives, such as mobile phones, iPads, television broadcasts, the Internet, and so on. Satellite communication systems are used on larger projects such as countries or Internet projects that work together.

Mobile communication technology is the first research and development direction in electronic communication technology. It is the largest and fastest technology. Since mobile communication can be augmented with increased wireless spectrum resources and wireless signals, this has caused traditional cellular communications to fall, and the scope of use has become increasingly limited. In addition, we have found many shortcomings in the research process, such as, now the reduction of cell radius makes the density of base stations gradually increase, frequent switching leads to waste of air resources and reduction of spectrum efficiency, which makes the network construction cost higher and higher. From the above shortcomings, we can see that in order to achieve higher spectral efficiency and larger and more sufficient capacity, we should break through the traditional communication system and explore new communication technologies.

\section{Key Technical Issues of Mobile Communication Systems}

In the mobile communication system, the current stage mainly adopts a distributed antenna approach to solve this problem. The processing unit of the plurality of wireless signals is disposed in the residential cell, and since the distance of the carrier wavelength is smaller than the propagation distance of the unit, the receiving and frequency conversion of the signal and the pre-processing can also be performed by these units. The processing function of the core unit is transmitted to the core of the processing unit after processing the information in the basic unit. When performing information processing, steps such as transceiving, frequency conversion, and pre-processing are required. Finally, the processing of information is achieved by coaxial fiber optic cable and fiber optic link. At present, there are two ways to implement the distribution. The first method is to simultaneously transmit the same downlink signal on the wireless signal processing unit, and then the wireless signal processing unit in the cell directly sends the uplink signal to the central processing unit after receiving the uplink signal [6]. The advantage of this scheme is that the operation method is simple and the learning time is short, but the disadvantage is that the signal transmission process will continuously interfere with the system, thereby hindering the expansion of the system capacity, which is not conducive to signal transmission. The second way is to complete the wireless full coverage distributed antenna structure throughout the service area. This method is implemented by a large number of wireless signal processing units, thus breaking the concept of the traditional cell. At the same time, this method can also be referred to as a "controlled antenna subsystem", that is, "only the signal processing unit close to the mobile station is responsible for communicating with the mobile station." In comparison, the second is more desirable than the first one, but it is more complicated to implement.

Distributed mobile communications have many advantages over traditional communication technologies. They are shown as following. First, the interval and interference is small, sir is high, and the system capacity is large. Second, the distributed internal partial set capability not only resists the shadow effect, but also ensures that the system capacity is not attenuated. Third, it can comprehensively improve its switching performance and receive signal power, reducing the number of switching. Fourth, under the same transmission power, the interference to other communication systems is small, the coverage area is large, and the transmission power is small. The five layers of the communication system are connected to each other and controlled separately. Only by thoroughly studying them can we better meet the needs of people's lives.

\section{Key Technical Issues of Satellite Communication Systems}

Satellite communication is a relatively mature technology developed in recent decades and is the most advanced technology in electronic communication technology. It also has the characteristics of strong anti-interference, large amount of transmitted information, and long distances and other technologies that are not available. However, in today's information age, people's craving for information is becoming more and more rapid, which puts higher requirements on the development 
of satellite communication systems.

At present, some key technologies of satellite communication still have some problems that need to be solved, including the demand for high-speed services and the IP difficulties of satellite communication applications. In order to solve these problems, in general, the key technologies of satellite communication generally have the following six points. First, the information is simply compressed. Whether it is dynamic or static information, the complexity of transmitting information is simplified, and the speed of information transmission is improved. Second, the transmission of information can be performed using a self-powered satellite antenna system. Third, improve the traditional IP selection method and adjust the broadband IP for designing satellite communication applications. Fourth, in order to simplify the complexity of the transmitted information, the transmission information can be processed by using a novel numerical modulation, coding improvement, and the like. Fifth, multiple addresses are processed in association with each other, which can reduce the problem of IP. Sixth is satellite laser communication technology, which can improve the processing efficiency of the system.

Future satellite communication technologies will be realized by data lasers, and the advantages of lasers will be fully utilized in Internet satellites. Because the application of laser communication technology has matured. Besides, the experimental data of scientists at any location can be fully proved, and some experiments are carried out in outer space, independent of the atmosphere. The use of lasers as carriers in space radio communications is promising for future satellite communications for laser communications.

\section{Conclusion}

In short, electronic communication systems have long been part of our daily lives, and the age of informationization has long since come. By analyzing the two most common technologies in electronic communication systems, we have learned and recognized electronic communication systems to identify problems and find solutions to better serve humans in the future.

The level of development of electronic communication systems directly determines the status and economic development of a country. Therefore, the analysis and research on the key technical issues of electronic communication systems is of paramount importance. Only by mastering its key technologies can it be better used and improved.

\section{Reference}

[1] K. Xu, Q. Cui and L. L. He. Key Issues and Solutions in Electronic Communication [J]. China Electronic Commerce, 2014 (20): 46.

[2] X. Yang. Analysis of Key Technical Issues of Electronic Communication System [J]. Wireless Interconnect Technology, 2013 (5):103.

[3] Y. C. Qi and L. P. Xu. Discussion on the Key Technical Issues of Electronic Communication Systems [J]. Information Systems Engineering, 2013 (8): 111.

[4] X. H. Peng. Analysis of the Key Technical Issues of Electronic Communication Systems [J]. Information Communication, 2013 (8): 178-179.

[5] Z. R. Guo. Analysis of the Key Technical Issues of Electronic Communication Systems [J]. Technology to Get Rich Guide, 2013 (15): 29.

[6] M. Li, C. Kuo and Y. M. Li. Discussion on Key Technical Issues of Electronic Communication System [J]. Science and Technology Outlook, 2015(6). 